\title{
Using the Properties of the Hyper Geometric Functions for Mona Lisa Original Image Detection
}

\author{
${ }^{1}$ Shaymaa Maki Kadham, ${ }^{2}$ Hind Rustum Mohammed, ${ }^{1}$ Shaymaa Abed Yasseen, \\ ${ }^{3}$ Hawraa Saheb Abo Hamed \\ ${ }^{1}$ Department of Mathematics, Faculty of Computer Sciences and Mathematics, \\ ${ }^{2}$ Department of Computer Sciences, Faculty of Computer Sciences and Mathematics, \\ ${ }^{3}$ Faculty of Computer Science and Mathematics, University of Kufa, Najaf, Iraq
}

\begin{abstract}
It is still a problem how to detection whether digital images are fake or original. In this study, a new algorithm has been developed that uses the properties of the hyper geometric functions to detect fake images from the original image of Mona Lisa by accurately analyzing all the image pixels and expanding the algorithm area by providing an experimental compensation of the $\mathrm{x}$ and $\mathrm{y}$ values and then reconstructing the regions and components and matching it to the original image. Statistical support for digital counterfeit image detection. The database used consisted of 15 fake images and the original image and the algorithm proved its efficiency accurately in detecting the forgery.
\end{abstract}

Key words: Finite symmetric, hyper geometric functions, orthogonally relation, transform linear, Mona Lisa, experimental compensation

\section{INTRODUCTION}

Image processing field concerned with the development and use of methods and algorithms forimages processing digital. Digitalimage encryption occupied a great deal of importance in the security and military fields (Taher and Mohammed, 2018).

An image is authentic if it represents a witness to an actual event place or time. A definition of image authenticity should enable us to distinguish an authentic image from the fake images in computer graphics images investigate the potential of global shape as a cue for object detection and recognition in computer vision (Schindler and Suter, 2008).

The Mona Lisa painting is an oil painting painted on a painting of wood painted by Leonardo da Vinci in 1503 and continued for many years until the completion of drawing. Some sources say it ended in 1501 and others say in 1510. Leonardo da Vinci began painting the Mona Lisa and officially finished a year. The painting's width was about $53 \mathrm{~cm}$ and its height was about $77 \mathrm{~cm}$ (Potter, 2006; Keith et al., 2011).

Hyper geometric functions (Barnard, 1999; Rakha et al., 2011; Wroughton and Cole, 2013): A hyper geometric function is defined as follows (Eq. 1 and 2):

$$
\frac{\Gamma(\mathrm{x}+\mathrm{r})}{\Gamma(\mathrm{x})} \text { and } \Gamma(\mathrm{x})=(\mathrm{x}-1) \text { ! }
$$

Special cases: if $y=z$

$$
\begin{aligned}
& F(x, y ; z ; G)=F(x, y ; y ; G)=\sum_{r=0}^{\infty} \frac{(X)_{r} \cdot G^{r}}{r !}=(1-G)^{-a} \\
& \operatorname{iFj}\left(x_{1}, x_{2}, x_{3}, x_{i} ; y_{1}, y_{2}, y_{j} ; G\right)=\sum_{r=0}^{\infty} \frac{\left(x_{1}\right)_{r}, \ldots,\left(x_{1}\right)_{r} \cdot G^{r}}{\left(y_{1}\right)_{r}, \ldots,\left(y_{1}\right)_{r} \cdot r !}
\end{aligned}
$$

such that: $\left(\mathrm{x}_{1}\right)_{\mathrm{r}}=$ through the numbers streptococcal will solve a single point when $|G|<1$ such that $G=1$ Eq. 3:

$$
\begin{aligned}
& \mathrm{G} \in[1, \infty) \text { for } \mathrm{x}=1 \text { then } \mathrm{F}(\mathrm{x}, \mathrm{y} ; \mathrm{y} ; \mathrm{G})= \\
& \sum_{\mathrm{r}=0}^{\infty} \mathrm{G}^{\mathrm{r}}=\frac{1}{1-\mathrm{G}}
\end{aligned}
$$

- Let $\mathrm{x}=2$ and $\mathrm{y}=1$ then $\mathrm{F}$ is called hyper geometric function

- And let $x=1$ and $y=1$ then $F$ is called kumfer function

\section{Theory 1:}

$$
\begin{aligned}
& 2 \mathrm{~F} 1(\mathrm{x}, \mathrm{y}, \delta, \mathrm{G})=\frac{\Gamma(\delta)}{\Gamma(\mathrm{y}) \Gamma(\delta-\mathrm{y})} \\
& \int_{0}^{1} \mathrm{t}^{(\delta \cdot \mathrm{y}-1)}(1-\mathrm{t})^{\mathrm{y}-1}(1-\mathrm{G})^{\mathrm{x}-1} \mathrm{dt}
\end{aligned}
$$

Theory 2:

$$
\begin{aligned}
& 2 \mathrm{~F} 1(\mathrm{x}, \mathrm{y}, \delta, 1)=\frac{\Gamma(\delta) \cdot \Gamma(\delta-\mathrm{x}-\mathrm{y})}{\Gamma(\delta-\mathrm{a}) \Gamma(\delta-\mathrm{y})} \\
& \text { for } \operatorname{Re}(\delta-\mathrm{a}-\mathrm{b})>0
\end{aligned}
$$

Corresponding Author: Shaymaa Maki Kadham, Department of Mathematics, Faculty of Computer Sciences and Mathematics, University of Kufa, Najaf, Iraq 
The relationship between hypergeometric function and other functions:

\section{1-Beta function:}

$$
\begin{gathered}
\mathrm{B}_{\mathrm{x}}(\mathrm{a}, \mathrm{b})=\frac{\mathrm{x}^{\mathrm{a}}}{\mathrm{a}} \cdot \mathrm{F}(\mathrm{a}, 1-\mathrm{a} ; 1+\mathrm{y} ; \mathrm{x}) \\
\mathrm{B}_{1}(\mathrm{a}, \mathrm{b})=\frac{\Gamma(\mathrm{a}) \cdot \Gamma(\mathrm{b})}{\Gamma(\mathrm{a}+\mathrm{b}) \Gamma(\delta-\mathrm{y})}
\end{gathered}
$$

Elliptic integers:

$$
\begin{aligned}
& Z(z)=\frac{\pi}{2} F\left(\frac{1}{2}, \frac{1}{2} ; 1 ; z^{2}\right) \\
& E(z)=\frac{\pi}{2} F\left(-\frac{1}{2}, \frac{1}{2} ; 1 ; z^{2}\right)
\end{aligned}
$$

\section{MATERIALS AND METHODS}

By applying the properties of hyper geometric functions and their relationship to some of the mathematical functions mentioned in this study. The phases of the proposed system, consisting of the following steps, Fig. 1 shows all phases of the proposed algorithm. The database contains an original image of Mona Lisa image and 15 counterfeit images to Mona Lisa image Fig. 2 illustrates the sample from the database.

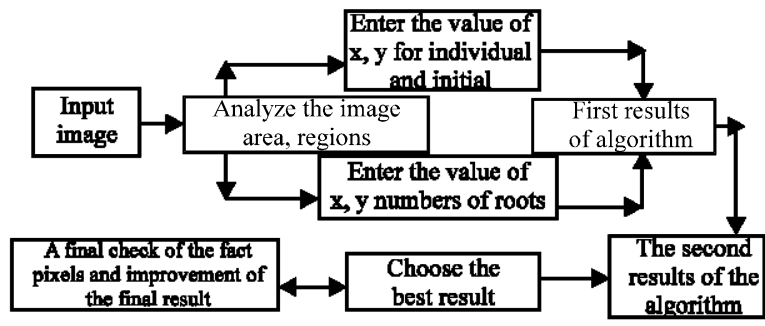

Fig. 1 : All phases of the proposed algorithm
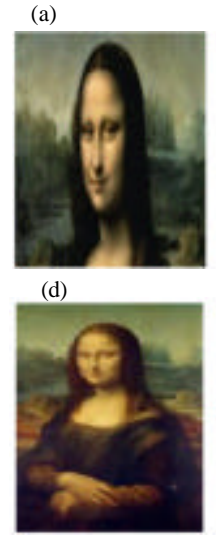

(b)
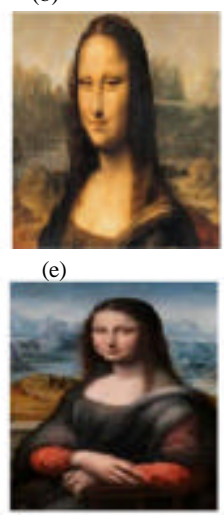

(c)
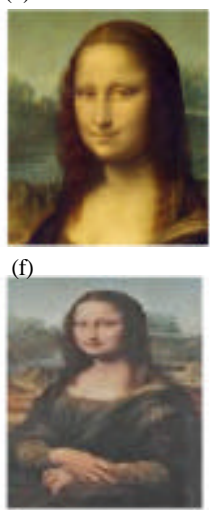

Fig. 2: a-f) Sample of the database used in the proposed method

\section{RESULTS AND DISCUSSION}

After the experimental application of the proposed system we found that there are after the features used by Leonardo da Vinci to draw Mona Lisa. They can be summarized as follows:

- The painting presents a woman in a half-body image

- Use the background of distant landscapes

- Use the simple description to clarify the meaning

- Use sensory sensory curves of incubator and clothing

Which were created through the progressive blending of the colors:

- Highlighting the forms of valleys and rivers behind women which gave the painting a three-dimensional character and contributed to clarify the details

- Drawing through the front and side together

- Use the blurry technique in drawing

- Use a set of colors interlaced with each other

- A technical approach to the perspective and especially in the background to make the background and not flatten

- Give a deep impression to make the picture more blurry and opaque whenever the distance of the viewer and increased depth

These features in the drawing make this painting a permanent record to see the painter. The 10 different differences were discovered between the fake images and the original image. Figure 3 illustrates the differences with the respective histograms for each variation with the original image regions.

In order to distinguish the fake image from the original image, we used the function of the algorithm in the detection of the falsification. The above function showed that the falsification by using some important properties where the compensation values to the variable $\mathrm{x}, \mathrm{y}$ with wild and preliminary numbers gave amazing results where the color blue represents the value of $x, y$ The initial individual numbers are 5 and 7 while the blue alona represents the root roots of root 2 and root 3 and the results showed differences in detection of falsification, thus, the image area readings will be very accurate. Figure 4 shows an illustration of the regions and the effect of the spread of false pixels and their dispersion through the paths of differences between the components of the original image and counterfeit images

Table 1 the values of the statistical parameters of the original picture are set while Table 2 shows the statistical parameters of the ten differences. 
J. Eng. Applied Sci., 14 (6): 1786-1792, 2019
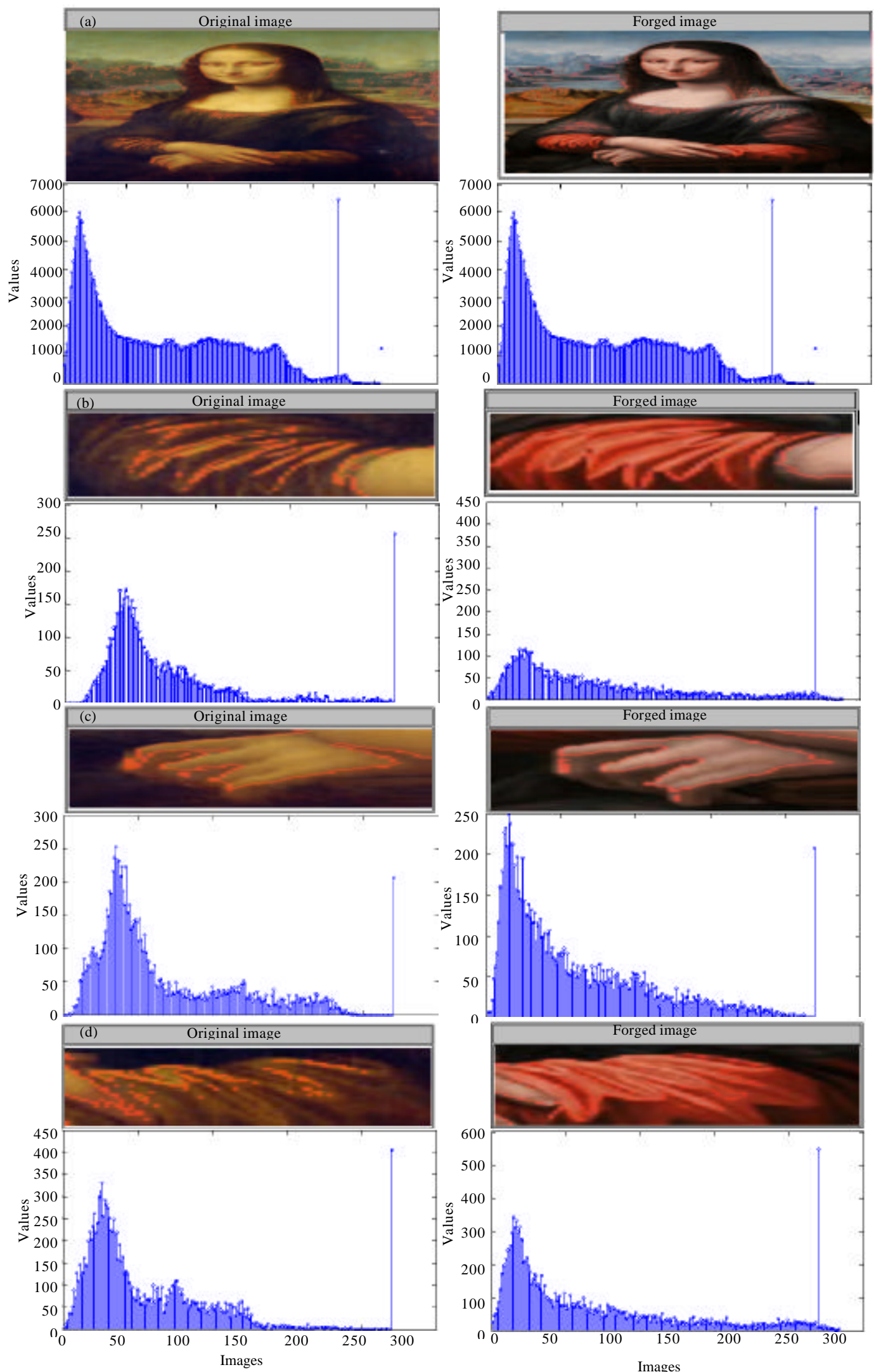

Fig. 3: Continue 

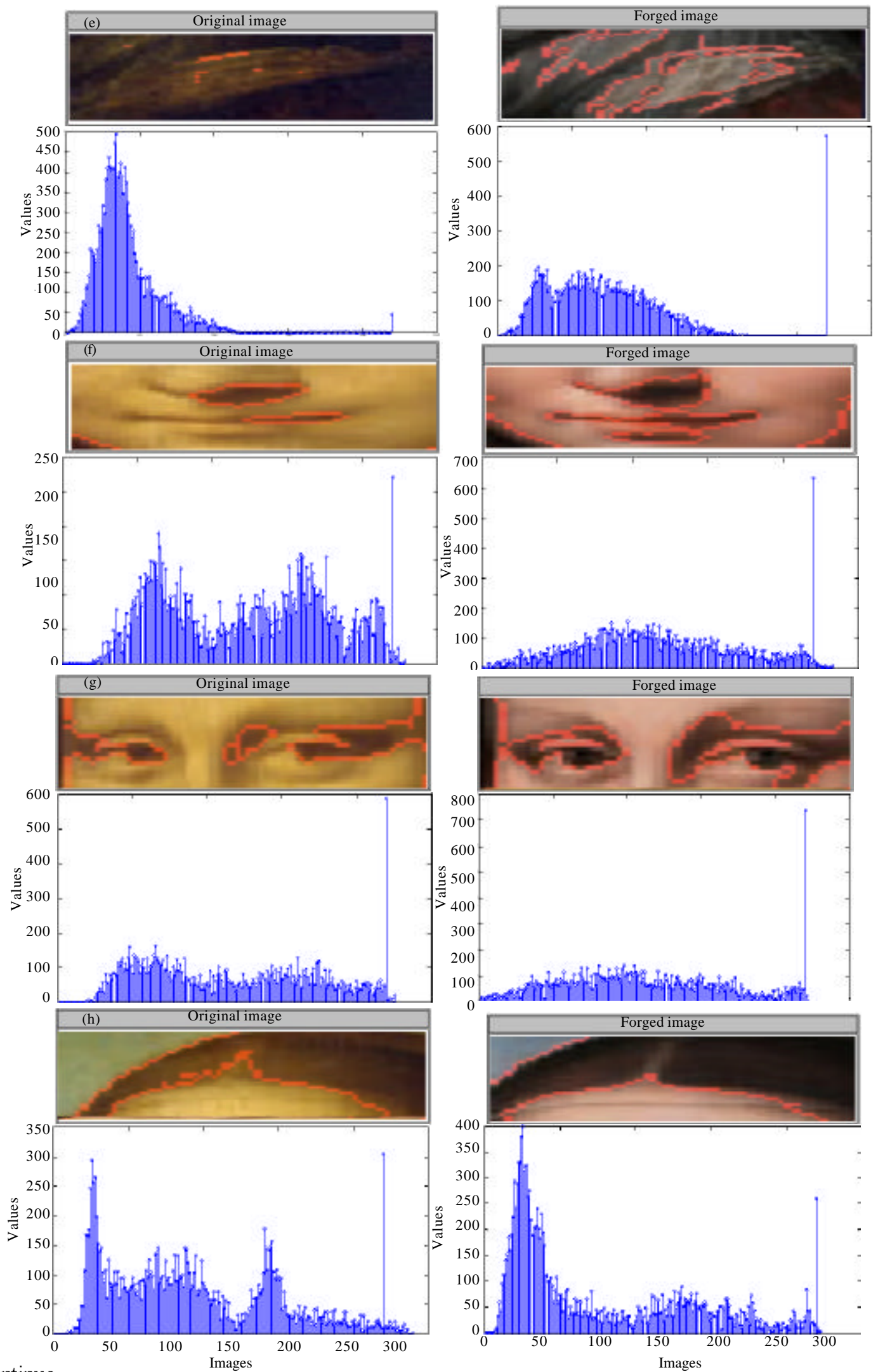

Fig. 3: Continue 
J. Eng. Applied Sci., 14 (6): 1786-1792, 2019
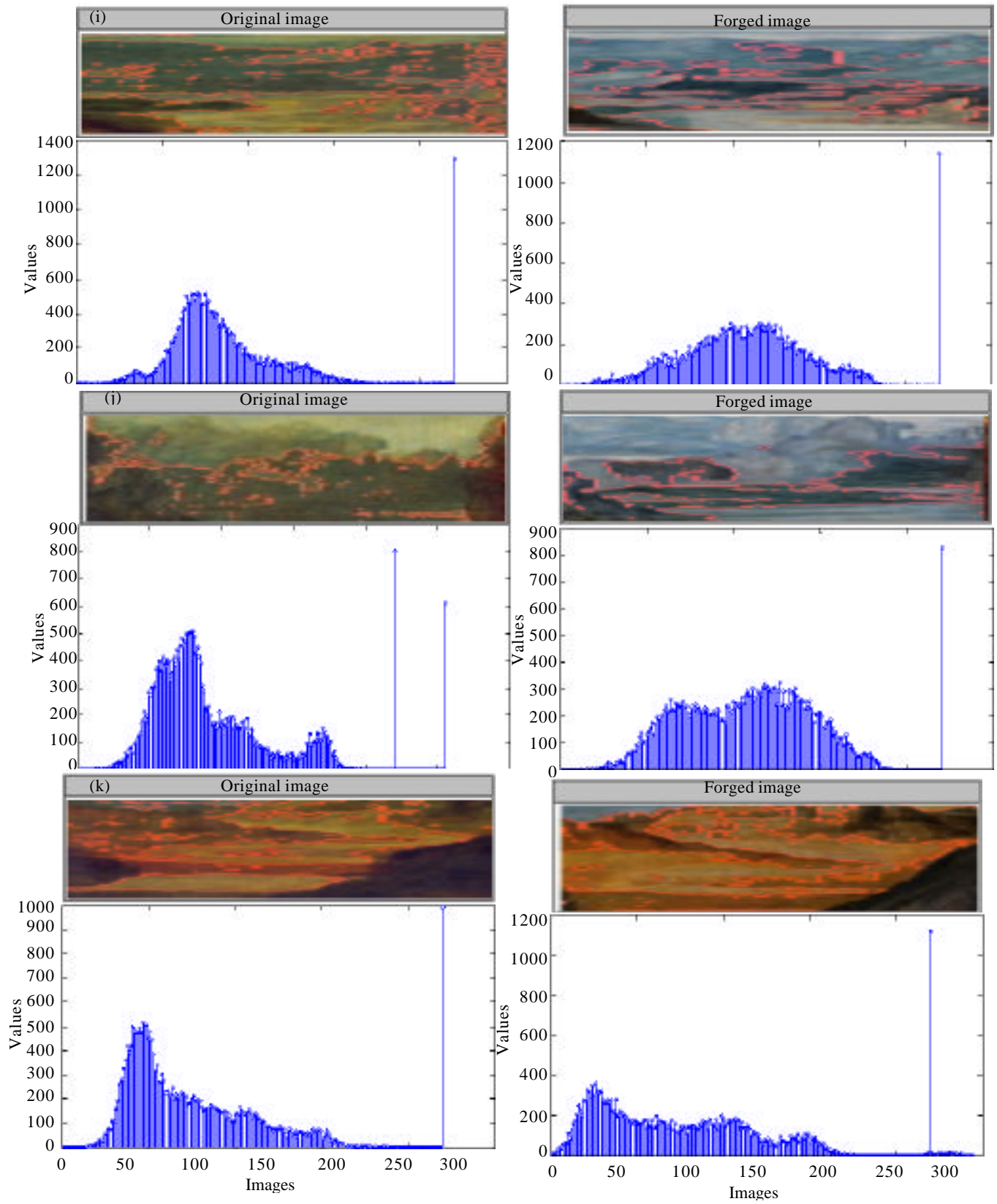

Fig. 3: Differences 1-10 with the respective histogram for each variation with the original image regions
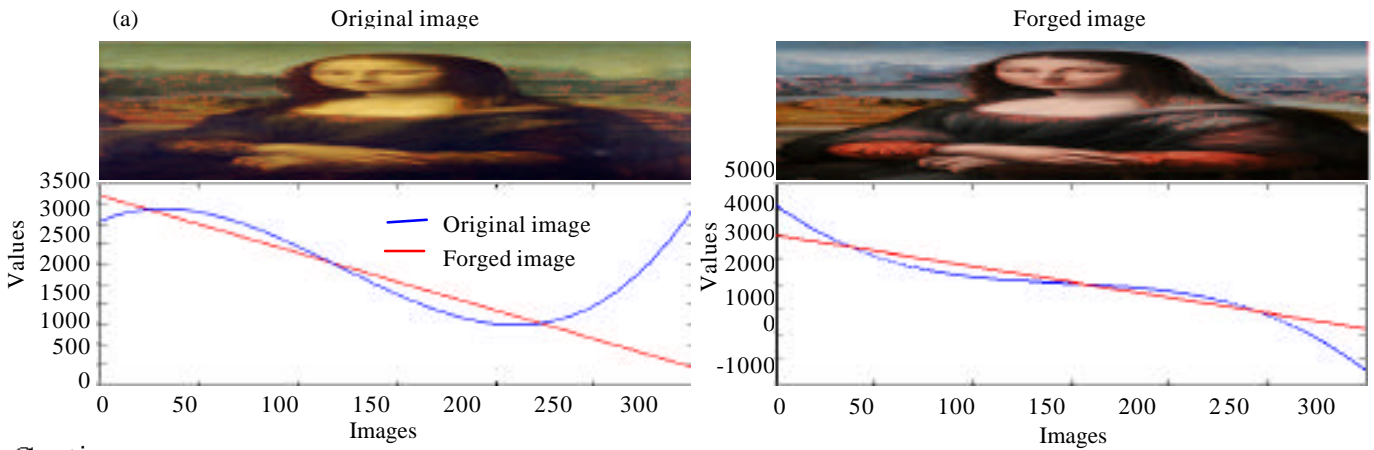

Fig. 4: Continue 
(b)

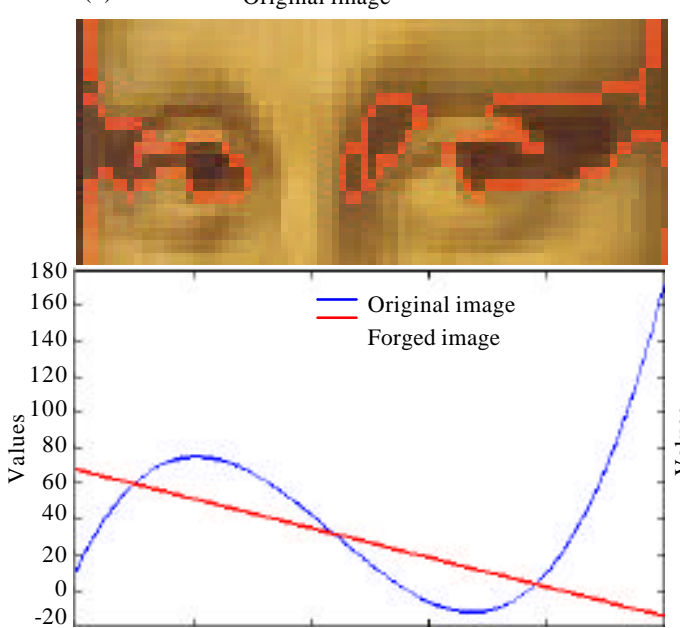

(c)

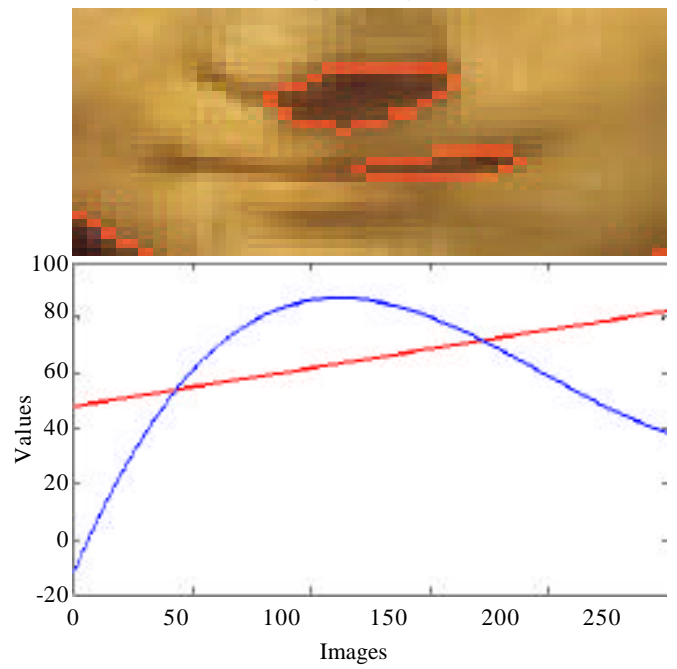

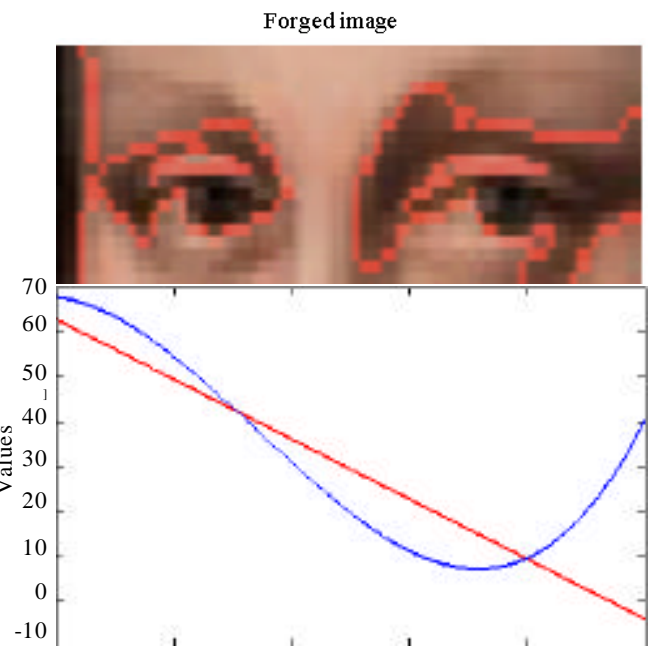

Forged image
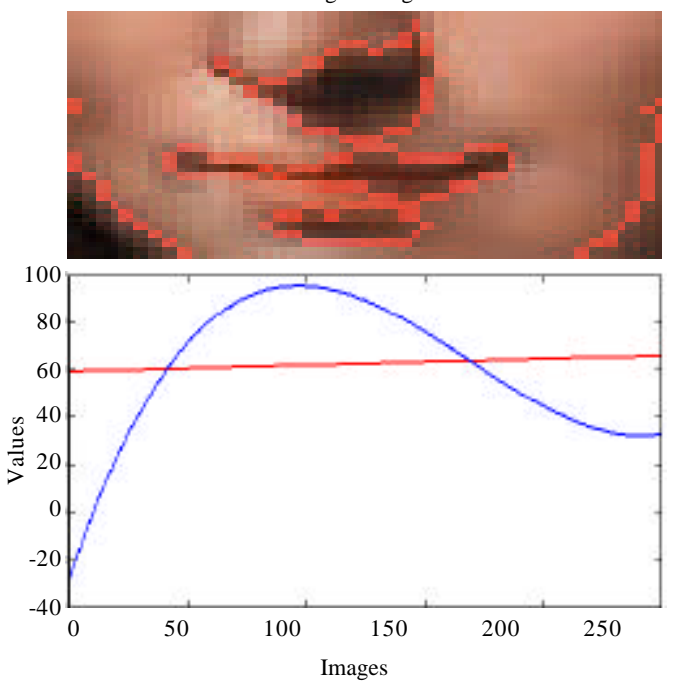

Fig. 4: a-c) Detection of region and the impact of the spread of false pixels and scattered between the components of the original image and fake images

Table 1: Values of the statistical parameters of the original image

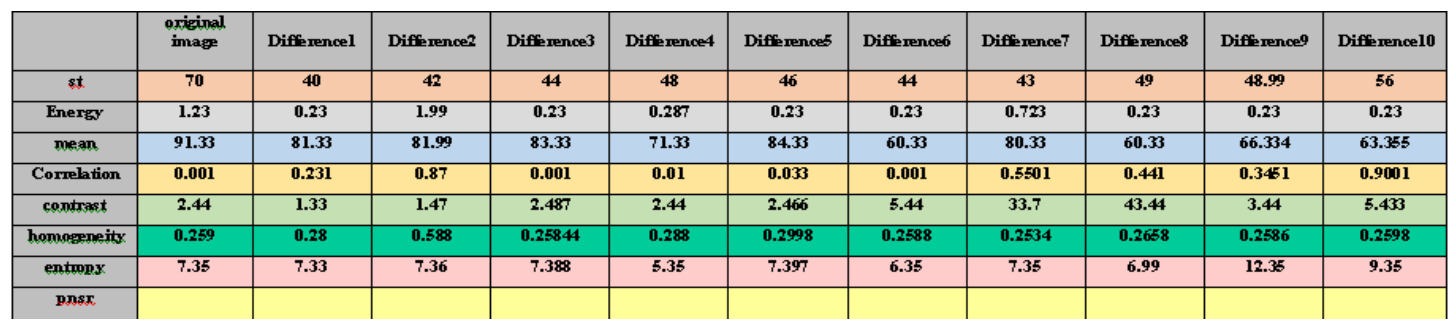

Table 2: Statistical characteristics of the ten difference of the fake image

\begin{tabular}{|c|c|c|c|c|c|c|c|c|c|c|c|}
\hline & Fake image & Differencel & Difference? & Difference3 & Difference4 & Differences & Difference6 6 & Difference? & Difference8 & Difference? & Differencel0 \\
\hline Energy & 1.32 & 0.32 & 2.99 & 4.32 & 0.32 & 0.32 & 0.32 & 0.32 & 0.32 & 0.32 & 0.345 \\
\hline Correlation & 0.02 & 0.332 & 0.762 & 0.06 & 0.02 & 0.432 & 4.02 & 4.02 & 4.02 & 4.112 & 7.78 \\
\hline contrast & 2.54 & 2.94 & 2.84 & 2.98 & 2.94 & 2.94 & 2.94 & 32.94 & 2.943 & 2.694 & 2.94 \\
\hline homogeneity & 0.025 & 0.0267 & 0.029 & 0.0280 & 0.024 & 0.088 & 0.0454 & 0.114 & 0.024 & 0.0414 & 0.0544 \\
\hline
\end{tabular}




\section{CONCLUSION}

The proposed method proved its efficiency in the accurate Mona Lisa original image detection by the properties of the hyper geometric functions. Results identified the following; the statistical parameters showed the importance of detecting the counterfeit images when mean in the original image and the parts of them starting 70 and decreasing up to 56 and the descent of values on a regular basis. In the fake image, the maen values are scattered. In some differences, high and low values appear. This indicates that the cropping of the fake image indicates a high difference in pixels compared to what is in the pixels of the original image.

Entropy in the original image ranges between 7.35-9.35 in the counterfeit image 7, 49-11, 49 and so on other statistical parameters as shown in Table 1 and 2 .

\section{REFERENCES}

Barnard, R.W., 1999. On applications of hypergeometric functions. J. Comput. Appl. Math., 105: 1-8.
Keith, L., A. Roy, R. Morrison and P. Schade, 2011. Leonardo da Vinci's Virgin of the rocks: Treatment, technique and display. National Gallery Tech. Bull., 32: $32-56$.

Potter, P., 2006. Art, science and life's enigmas. Emerging Infect. Dis., 12: 1308-1309.

Rakha, M.A., A.K. Rathie and P. Chopra, 2011. On some new contiguous relations for the Gauss hypergeometric function with applications. Comput. Math. Appl., 61: 620-629.

Schindler, K. and D. Suter, 2008. Object detection by global contour shape. Pattern Recognit., 41: 37363748.

Taher, S.A. and H.R. Mohammed, 2018. Combination mathematical distance measure approach for some image processing applications. J. Theor. Appl. Inf. Technol., 96: 2169-2183.

Wroughton, J. and T. Cole, 2013. Distinguishing between binomial, hypergeometric and negative binomial distributions. J. Stat. Educ., 21: 1-16 